Original Research Paper

\title{
Effect of Di-(2-Ethylhexyl) Phthalate on the Microbial Community in Vegetables Phyllosphere
}

\author{
${ }^{1,2}$ Xiaowei Fu ${ }^{*}$ and ${ }^{3}$ Dan $\mathrm{Wu}$ \\ ${ }^{1}$ College of Life Science, University of China Jiliang, Zhejiang, Hangzhou, 310018, China \\ ${ }^{2}$ Zhejiang Provincial Enineering Laboratory of Quality Controlling Technology and Instrumentation for Marine Food, China \\ ${ }^{3}$ Department of Food Science and Nutrition, Zhejiang University, Hangzhou, 310022, China
}

\author{
Article history \\ Received:12-10-2019 \\ Revised: 02-01-2020 \\ Accepted: 10-01-2020 \\ Corresponding Author: \\ Xiaowei Fu \\ College of Life Science, \\ University of China Jiliang, \\ Zhejiang, Hangzhou, 310018 , \\ China \\ Fax: 0571-8767-6372 \\ Tel: 0571-8767-6372 \\ Email: mail:fxw13@163.com
}

\begin{abstract}
A panel count method and a phospholipid fatty acid analysis technique were used to investigate the responses of Di-(2-Ethylhexyl) Phthalate (DEHP) pollutants to the phyllosphere microbial communities of Chinese Cabbage. The results of plate count analysis showed that the number of culturable microorganisms could be lower than that of water spray after treated with a certain amount of DEHP pollutant. The results of phospholipid fatty acid analysis showed that DEHP pollution treatment had certain effects on the biomass and community structure of leaf microorganisms in leaves of Chinese Cabbage. After spraying 1d, the biomass of the total leaf microorganisms, fungi and bacteria in the leaves of vegetables decreased, which was significantly different from that in the control and the blank groups. There was little change in the ratio of the characteristic phospholipid fatty acids between gram positive bacteria and gram negative bacteria.
\end{abstract}

Keywords: DEHP, Phyllosphere, Microbial Community

\section{Introduction}

Aerial plant surfaces are generally colonized by large numbers of different microorganisms including bacteria, filamentous fungi, yeasts and algae. The aerial habitat colonized by these microbes is termed as phyllosphere and the inhabitants are called phyllosphere microbes (Lindow and Brandl, 2003). Microorganisms in the phyllosphere may include those that are pathogenic to the plant and nonpathogenic organisms, which play an important role in altering plant surface properties, fixating nitrogen, promoting the growth of plants, controlling the plant pathogens, degrading organic pollutants and being an ideal monitors of plants pollution (Krechel et al., 2002; Krimm et al., 2005; Kvasnikov et al., 1974; Sandhu et al., 2007; Schreiber et al., 2005).

Vegetable is an indispensable food in people's daily life and the quality of vegetables has a close relationship with human health. In recent years, more and more attention has been paid to the pollution of vegetables by Di-(2-Ethylhexyl) Phthalate (DEHP) with the continuous development of plastic film and greenhouse. DEHP is one of the most widespread phthalate plasticizer, used in numerous consumer products, commodities, medical devices, food packaging and building materials (Jing et al., 2010; Zhang et al., 2009a; Wang et al., 2008; Sablayrolles et al., 2005). As to health effects from human exposure to DEHP, DEHP is teratogenic, mutagenic and carcinogenic and the EPA of USA has classified them as priority pollutants (Pandey and Chauhan, 2007; Sandhu et al., 2007). Because of the large and widespread application, DEHP has been widely detected in food, air, water, soil and sediments (Schreiber et al., 2005; Yang et al., 2001). In our previous studies, we found that vegetables grown in plastic film and plastic industrial areas mainly absorbed DEHP in the atmosphere through the leaves or above ground parts and accumulated to the stems, fruits and roots, which eventually led to the pollution of vegetables by DEHP. Studies have shown that microorganisms in plant leaves have the ecological function of degrading organic molecules. Therefore, we would like to use vegetable leaf microorganisms to reduce DEHP pollution in the atmosphere, thus blocking DEHP into vegetables. The aim of this study was to investigate the responses of DEHP pollutants to the phyllosphere microbial communities of Chinese Cabbage.

\section{Materials and Methods}

\section{Experimental design}

On June 2015, Brassica chinensis var chinensis are potted in the sun room. For about 1 weeks, Brassica chinensis var chinensis grew about $7 \mathrm{~cm}$ at first and then 
leaf surface began to be sprayed with DEHP. The concentrations of $10 \%$ and $80 \%$ DEHP were designed for the treatment group. Leaf spray as a contrast and leaves without any processing were blank. The experiments were divided into 3 sprays, DEHP (labeled I II III, every 6d spray 1 times). And the experiment lasted 3 weeks. After 1,3,7d DEHP, samples such as leaves and stems were collected at random (each group was collected about $100 \mathrm{~g}$ ). The samples were collected and placed in a sterile sample bag to be taken back to the laboratory for direct analysis.

\section{Microorganism Cell Extraction}

The $10 \mathrm{~g}$ sample was treated with $100 \mathrm{~mL}$ phosphate buffer solution ( $\mathrm{pH} 7.0$, containing $0.1 \%$ Tween 20). After ultrasonic oscillation (40 KHZ) treatment of $10 \mathrm{~min}$, the sample was shaking for $50 \mathrm{~min}$ at $25^{\circ} \mathrm{C}$, the 200 r.min ${ }^{-1}$. At last, microorganisms were eluted from the leaves, ans the lotion was reserved.

\section{Microbial Culture and Enumeration}

Different media were prepared: Vegetable juice, solid medium and LB solid medium. Take appropriate elution dilution of $1 \mathrm{ml}$, respectively, with the above three kinds of medium for casting plate colony culture. The number of colonies was counted after $3 \mathrm{~d}$ $5 \mathrm{~d}$ at $30^{\circ} \mathrm{C}$ constant temperature.

\section{Phospholipid Fatty Acid Analysis (PLFAs)}

The collected vegetable phyllospheric microorganism was precipitated and the total esters were extracted by chloroform, methanol and phosphate buffer solution. And then phospholipid fatty acid was isolated by silica gel column chromatography. A esterified solution was dissolved in a positive nineteen alkane fatty acid methyl ester containing $33 \mathrm{~g} / \mathrm{mL}$ internal standard volume ratio 1:4 of chloroform: hexane the solvent, dried by nitrogen and then detected by GC-MS.

The biomass of phyllosphere bacteria was estimated by the total PLFA of 14:0, 15:0, 16:0, 18:0, i15:0, i16:0, i17:0,

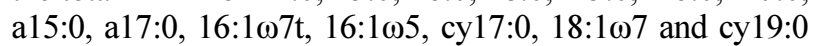
(Lindow and Leveau, 2002; Zhang et al., 2008; Wang et al., 2010; Song et al., 2010). The biomass of fungi was

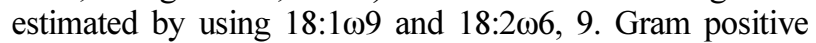
bacteria content was estimated using i15:0, i16:0, i17:0,

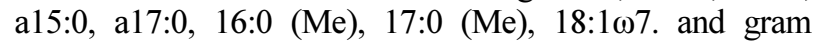
negative bacteria by $16: 1 \omega 5,16: 1 \omega 7 \mathrm{t}, 16: 1 \omega 9$, cy17:0, $18: 1 \omega 5,18: 1 \omega 7$, cy 19:0.GP/GN represents the ratio of gram positive and gram negative bacteria biomass

\section{Data Analysis}

The count of culturable microorganisms was counted by plate count method. Fatty acids with carbon lengths of 14 to 20 were used to analyze the microbial community structure of the phyllosphere. Data were presented as means together with Standard Deviations (SDs). For PLFA concentrations, the ratios of the different microbial groups, in addition to the GP/GN, were analyzed using Analysis Of Variance (ANOVA) with SPSS software.

\section{Results and Discussion}

\section{The Number of Microorganisms in the Leaves of Different Treatment Samples can be Cultured}

Vegetable Juice Solid Medium was Used to Count the Number of Culturable Microorganisms in the Leaves of Vegetables

The number of culturable microorganisms in the leaves of vegetables was counted by plate count method, in which the number of fungi was mainly counted in the solid medium of vegetable juice. From the panel point of view, the number of microorganisms in the solid medium of vegetable juice solid was $\left(10^{3}-10^{4}\right) \mathrm{cfu} / \mathrm{g}$ sample. As shown in Fig. 1.

The experiment was divided into 3 sprays DEHP (I, II, III) respectively. The treatments were: spraying $10 \%$ concentration of DEHP, $80 \%$ concentration of DEHP, spraying water and blank control without any treatment. As shown in Fig. 1, the 0d sample (blank control, spraying water, $10 \%$ concentration and $80 \%$ concentration) were considered as the blank sample, because of all the treatment before sampling and culture counting, from four samples of vegetables $0 \mathrm{~d}$, each microorganism quantity had little difference. Compared with the sample of $0 \mathrm{~d}$, the amount of bacteria in 1d blank and control samples increased, while the quantity of bacteria in 1d DEHP samples was lower than that of $0 \mathrm{~d}$. Compared with spraying $10 \%$, the concentration of $1 \mathrm{~d}$ in the $80 \%$ samples decreased obviously compared with that of $0 \mathrm{~d}$. That the number of microorganism with time and environment showed dynamic changes and after spray and spray DEHP treatment has obvious influence on the vegetables phyllospheric microorganism, spray DEHP is likely to inhibit some of DEHP sensitive microbial growth and reproduction and it is good for some water like wet microbial growth and reproduction. Overall, the number of culturable microorganisms in DEHP samples was lower than that in controls and blanks. In addition, in the whole experiment process, from the beginning of $0 \mathrm{~d}$ to the end of the third spraying, the amount of bacteria in the blank and control samples has been on the rise. Except for the III-2 of the blank sample, the amount of bacteria has dropped somewhat. It can be seen from the picture: three times of spraying DEHP, spraying $80 \%$ concentration of DEHP samples of vegetables, the number of microorganism was significantly higher than that of spraying $10 \%$ concentration is low; microorganism quantity of spraying DEHP on the first day after the sample was third, 6D small samples, it might just spraying DEHP, DEHP changed the leaves international environment, thus affecting the sensitivity of 
microbial growth and reproduction, with the extension of time, some microbes might adapt to existing phyllospheric environment DEHP, DEHP added in the highly volatile environment, there may be the concentration of DEHP is more and more low, phyllosphere microorganisms will no longer cause effect.

\section{LB Solid Medium Counts the Number of Culturable Microorganisms in the Leaves of Vegetables}

The number of culturable microorganisms in leaves of vegetables was counted by plate count method and the number of bacteria in LB solid medium was mainly counted. From the panel point of view, the number of microorganisms in the LB solid medium was $\left(10^{4}-10^{5}\right)$ cfu/g samples.

As shown in Fig. 2 and the 0d sample (blank control, $10 \%$ concentration and $80 \%$ concentration) were considered as the blank sample, because of all the treatment before sampling and culture counting, from four samples of vegetables $0 \mathrm{~d}$, each microorganism quantity had little difference. In the whole experiment process, from the beginning of $0 \mathrm{~d}$ to the third spraying, the amount of bacteria in the blank and control samples has been increasing. Except for the II-2 of the control samples, the amount of bacteria has dropped slightly. Three times of spraying DEHP, spraying $80 \%$ concentration of DEHP samples of vegetables, the number of microorganism was significantly higher than that of spraying $10 \%$ concentration is low; microorganism quantity of spraying DEHP on the first day after the sample was third $6 \mathrm{D}$, the sample should be less. In addition, we also found that phyllospheric microorganism $80 \%$ DEHP treated samples compared with the vegetables vegetable juice phyllospheric microorganism culture medium and the vegetable juice culture medium for fungi, while LB medium is more suitable for bacteria, therefore, phyllosphere microbes not only how much training and do processing and related the choice of the medium etc.

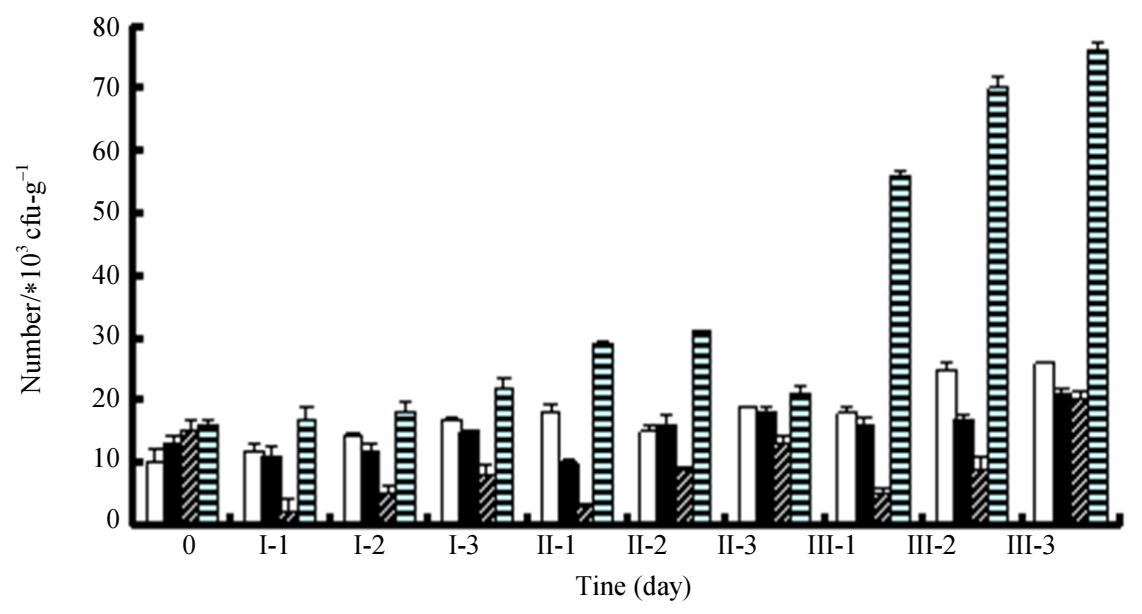

Fig. 1: Effects of DEHP on the number of culturable microorganisms in Brassica chinensis var chinensis (The columns were left to right: $\mathrm{CK}, 10 \%$ concentration, $80 \%$ concentration, blank), vegetable juice served as a medium

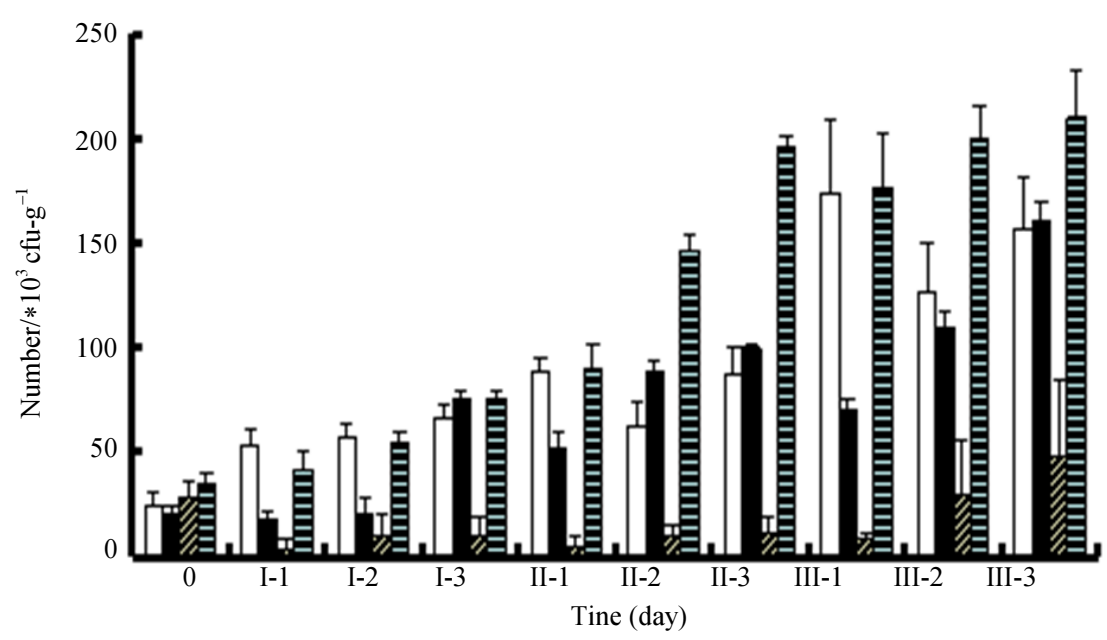

Fig. 2: Effects of DEHP on the number of culturable microorganisms in Brassica chinensis var chinensis (The columns were left to right: $\mathrm{CK}, 10 \%$ concentration, $80 \%$ concentration, blank), LB solid medium served as a medium 
From the plate, vegetable juice solid medium fungi order $\left(10^{3}-10^{4}\right) \mathrm{cfu} / \mathrm{g}$ samples, LB bacterial culture order $\left(10^{4}-10^{5}\right) \mathrm{cfu} / \mathrm{g}$ samples, may be a solid vegetable juice culture medium is LB medium lack of nutrition training in vegetable juice is more suitable for fungi, so two kinds of culture medium statistical microorganisms the magnitude of difference.

\section{Phosphate Fatty Acid Analysis (PLFAs) Results}

Almost all microbial membranes contain phospholipids, which are relatively constant. Different microorganisms contain different kinds and amounts of PLFAs, some of which are specific to PLFAs and exist in certain microbial cell membranes. Thus, PLFAs can be used as a biomarker for microbial biomass and community structure change in (Zhang et al., 2009b; Wang et al., 2008; Sablayrolles et al., 2005).

This experiment identified 18 PLFAs, with carbon chains ranging in length from 15 to 20 , including saturated, unsaturated, propane and methyl fatty acids. Figure $3 \mathrm{a}$ shows the dynamic change of total PLFAs content in the leaves of the vegetables during the experiment. There is a significant difference in the content of PLFAs between DEHP contaminated vegetables and water samples. With the increase of time, the total content of PFLAs in water samples slowly increased, while the total PLFAs content in vegetables samples DEHP spraying treatment were lower than the water sample, indicating that DEHP pollutants have a certain influence on soil microbes. The total content of PLFAs in vegetable samples three times spraying after DEHP treatment, the first measured decreased the content of first times the 1D samples to reduce the magnitude of the maximum, the content of 1D after two times of treatment by measuring the decrease trend is relatively slow and then as time changes, the both contents of PFLAs in $3 \mathrm{~d}$ and $7 \mathrm{~d}$ were gradually increased, which can explain the vegetables phyllospheric microorganism has a process of adaptation to DEHP, but also that certain microorganisms may produce a metabolic ability to DEHP. In addition DEHP with the extension of treatment time, some microorganisms have begun to accelerate growth and reproduction, so we infer that some phyllospheric microorganisms may be key to DEHP as the carbon source and their nutrition to promote its rapid growth and reproduction, performance for the total increase in the content of FLFAs.

Phospholipid fatty acids $(18: 1, \omega 9)$ and $(18: 2, \omega 6,9)$ were the main indicators of fungi and the content of these two fatty acids was used to estimate fungal PLFAs content. Figure $3 \mathrm{~b}$ and $3 \mathrm{c}$ showed that the PLFAs content of fungi and bacteria treated by water spraying was higher than that by spraying DEHP. With the change of time, phyllosphere fungi and bacteria in water treatment are gradually increased and the content of the 1D were fungi every treatment spray DEHP decreased significantly, then began to increase, third of which sprayed third times the content of DEHP increased rapidly after fungi. For the DEHP bacteria, the dynamic changes of PLFAs content were slightly more gentle than those of fungi, which indicated that DEHP pollutants had a great influence on fungi.

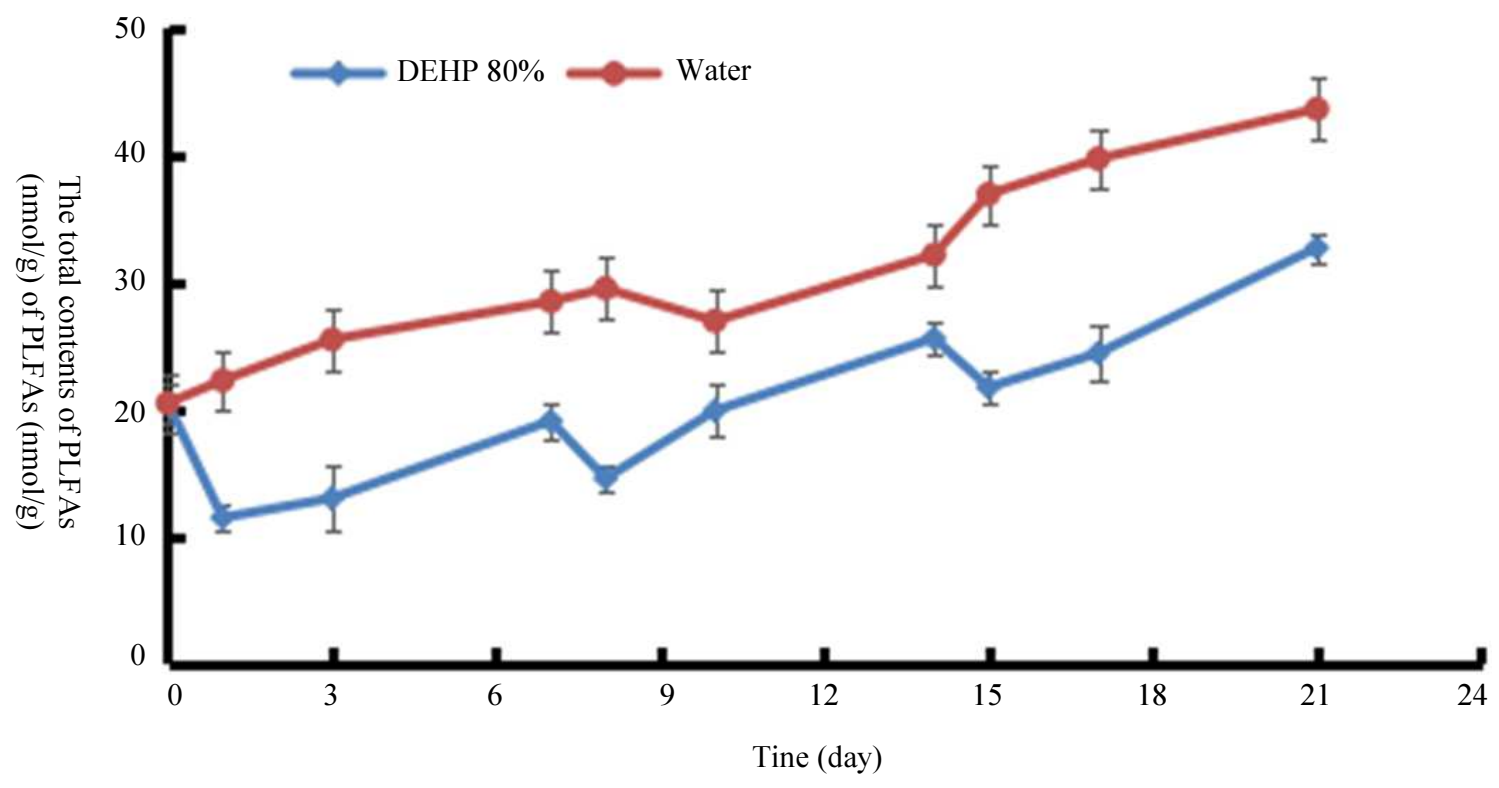

(a) 


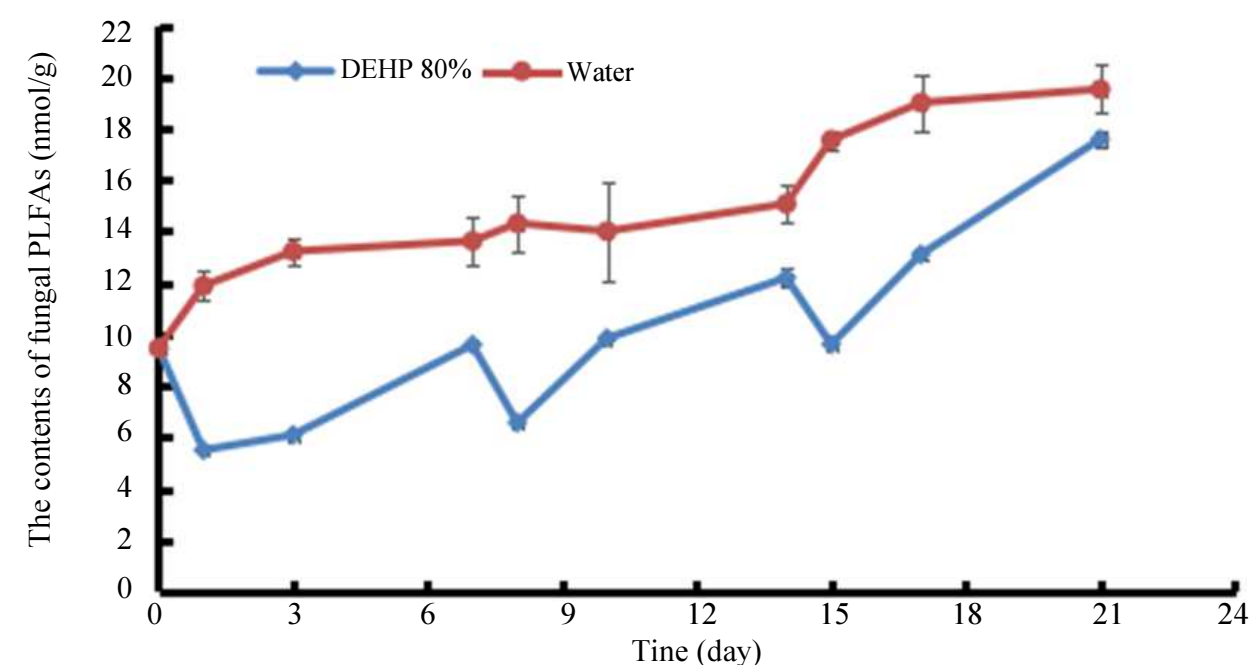

(b)

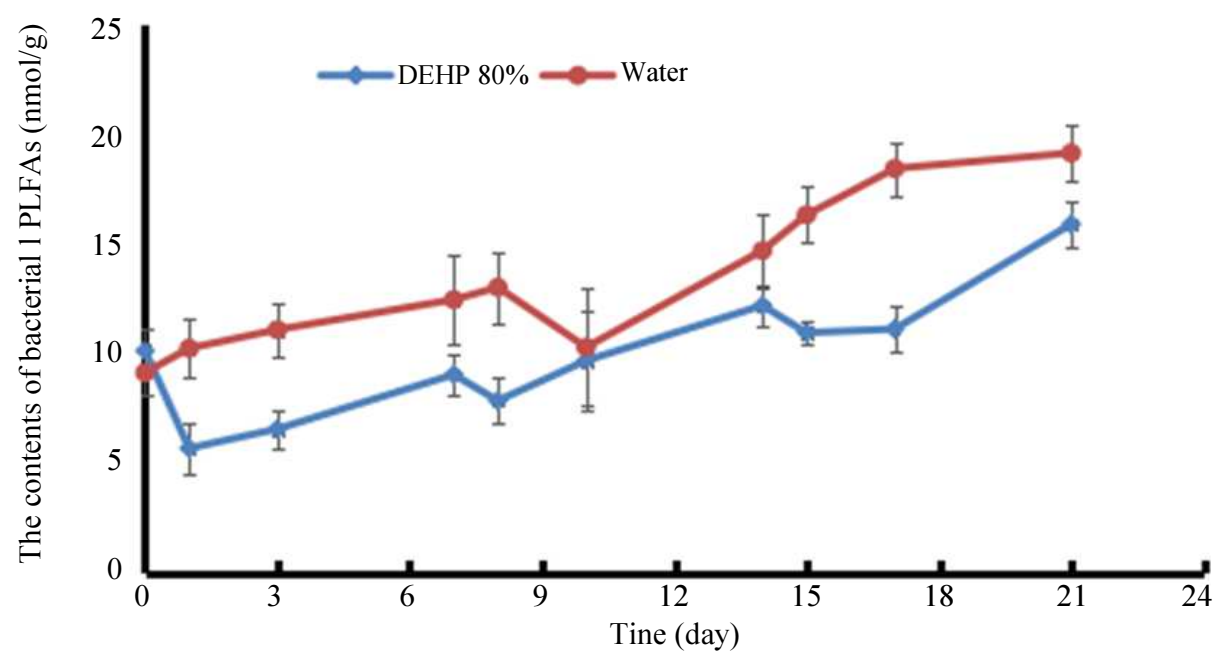

(c)

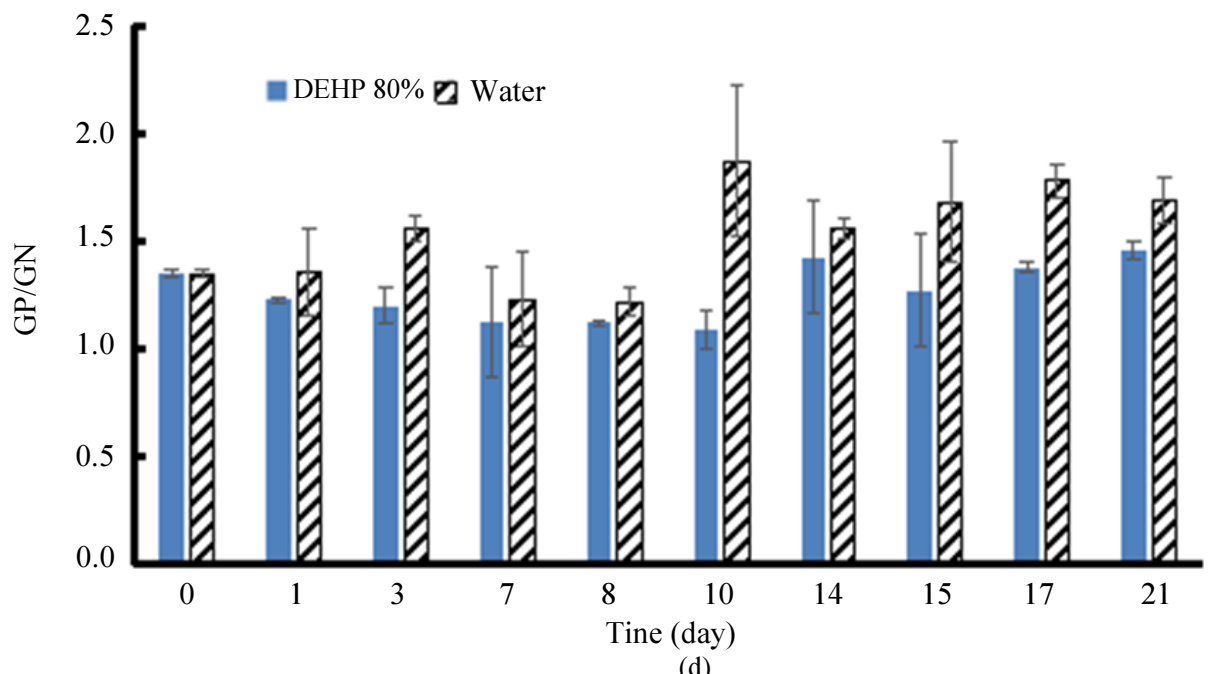

Fig. 3: Twenty-one-day response of (a) total microbial fatty acids, (b) fungal fatty acids, (c)bacterial fatty acids and (d)GP/GN PLFA to DEHP in Brassica chinensis var chinensis 
The contents of gram positive and negative bacteria in leaf microorganisms of DEHP and GP/GN treated plants were analyzed, as shown in Fig. 3d. As shown, different treatments affect the levels of gram positive and negative bacteria. For water treatment, the ratio of GP/GN has a phase change, in weeks, in the week, the content change increased, indicating that during this period the gram positive bacteria growth rate faster than gram negative bacteria. For the injection of DEHP treated samples, GP/GN ratio decreased first and then increased slowly, the ratio were 1D every time the decline, then began to increase, perhaps because of gram negative bacteria with DEHP can be degraded and can convert DEHP to their nutrient substance use, promote the growth and reproduction. There may be DEHP environmental pollutants have certain inhibitory effect on Gram negative bacteria, with the passage of time, gram negative bacteria began to adapt to DEHP, have a certain resistance to DEHP toxicity and slowly growth reproduction. Therefore, according to the indicator of gram negative bacteria, phospholipid fatty acids, which we can use to judge and investigate the pollution levels of environmental pollutants DEHP.

\section{Conclusion}

We found that the number of culturable microorganisms could be lower than that of water spray after treated with a certain amount of DEHP pollutant by the plate count. And some results showed that DEHP pollution treatment had certain effects on the biomass and community structure of leaf microorganisms in leaves of Chinese Cabbage. After the one-day treatment, the biomass of the total leaf microorganisms, fungi and bacteria in the leaves of vegetables decreased, which was significantly different from that in the control and the blank groups. What's more, there was slightly change in the ratio of the characteristic phospholipid fatty acids between gram positive bacteria and gram negative bacteria. These results may be helpful to judge and investigate the pollution levels of environmental pollutants DEHP.

\section{Acknowledgement}

This research was supported by a fund (LY16D010003) from the Zhejiang Provincial National Science Foundation of China.

\section{Author's Contributions}

Xiaowei Fu: Participated in all experiments, coordinated the data-analysis and contributed to the writing of the manuscript.

Dan Wu: Coordinated the data-analysis.

\section{Conflict of Interest}

The author (Xiaowei Fu) had no conflict of interest to declare.

\section{References}

Jing, M., Y.M. Su, L.K. Gu, X.W. Peng and L. Li et al., 2010. Effects of dichlorovos insecticide on the microbial community in peach phyllosphere. J. Agro-Environ. Sci., 29: 864-869.

Krechel, A., A. Faupel, J. Hallmann, A. Ulrich and G. Berg, 2002. Potato-associated bacteria and their antagonistic potential towards plant-pathogenic fungi and the plant-parasitic nematode Meloidogyne incognita (Kofoid and White) Chitwood. Canadian J. Microbiol., 48: 772-786. DOI: 10.1139/w02-071

Krimm, U., D. Abanda-Nkpwatt, W. Schwab and L. Schreiber, 2005. Epiphytic microorganisms on strawberry plants (Fragaria ananassa cv. Elsanta): Identification of bacterial isolates and analysis of their interaction with leaf surfaces. FEMS Microbiol. Ecol., 53: 483-492.

DOI: 10.1016/j.femsec.2005.02.004

Kvasnikov, E.I., T.M. Kliushnikova and S.S. Nagornaia, 1974. The nitrogen fixing ability of yeasts isolated from the rhizosphere and phyllosphere of plants. Microbiology, 36: 790-791.

Lindow, S.E. and J.H. Leveau, 2002. Phyllosphere microbiology. Curr. Opi. Biotechnol., 13: 238-243. DOI: 10.1016/S0958-1669(02)00313-0

Lindow, S.E. and M.T. Brandl, 2003. Microbiology of the phyllosphere. Applied Environ. Microbiol., 69: 1875-1883.

DOI: 10.1128/AEM.69.4.1875-1883.2003

Pandey, P. and U.K. Chauhan, 2007. Effects of pesticides (endo sulfan and quinal phos) on microbial community under controlled conditions. FASEB J., 21: 791-792.

Sablayrolles, C., M. Montrejaud-Vignoles, D. Benanou, L. Patria and M. Treilhou, 2005. Development and validation of methods for the trace determination of phthalates in sludge and vegetables. J. Chromatography A, 1072: 233-242. DOI: 10.1016/j.chroma.2005.02.074

Sandhu, A., L.J. Halverson and G.A. Beattie, 2007. Bacterial degradation of airborne phenol in the phyllosphere. Environ. Microbiol., 9: 383-392. DOI: $10.1111 /$ j.1462-2920.2006.01149.x

Schreiber, L., U. Krimm, D. Knoll, M. Sayed and G. Auling et al., 2005. Plant-microbe interactions: Identification of epiphytic bacteria and their ability to alter leaf surface permeability. New Phytol., 166: 589-594.

DOI: $10.1111 / \mathrm{j} .1469-8137.2005 .01343 . \mathrm{x}$ 
Song, G.Y., J.Y. Dai and F. Hu, 2010. Accumulation of phthalic acid esters in different types of soil-plant systems. J. Agro-Environ. Sci., 29: 1502-1508.

Wang, P., S.L. Wang and C.Q. Fan, 2008. Atmospheric distribution of particulate- and gas-phase Phthalic Esters (PAEs) in a Metropolitan City, Nanjing, East China. Chemosphere, 72: 1567-1572. DOI: 10.1016/j.chemosphere.2008.04.032

Wang, S.J., Y.Q. He and S. Ye, 2010. Effects of Di-(2ethylhexyl)Phthalate (DEHP)on POD activities and MDA contents in stem and leaves of vicia faba seedlings. Asian J. Ecotoxicol., 5: 587-591.

Yang, C.H., D.E. Crowley, J. Borneman and N.T. Keen, 2001. Microbial phyllosphere populations are more complex than previously realized. Proc. Natl Acad. Sci. USA, 98: 3889-3894.

DOI: $10.1073 /$ pnas.051633898
Zhang, B.G., H.X. Zhang and B. Jin, 2008. Effect of cypermethrin insecticide on the microbial community in cucumber phyllosphere. J. Environ. Sci., 20: 1356-1362. DOI: $10.1016 / \mathrm{S} 1001-0742(08) 62233-0$

Zhang, B.G., L. Tang and Z.M. Li, 2009a. Effect of abamectin insecticide on the microbial community in broccoli phyllosphere. Environ. Sci., 30: 1292-1297.

Zhang, B.G., Z.H. Bai and L. Tang, 2009b. The impacts of cypermethrin pesticide application on the nontarget microbial community of the pepper plant phyllosphere. Sci. Total Environ., 407: 1915-1922. DOI: $10.1016 /$ j.scitotenv.2008.11.049 